\title{
Performance Analysis of Scheduling Strategies in LTE based on Open-Loop Spatial Multiplexing MU-MIMO Transmission Mode
}

\author{
Ibrahim A. Alfatah Ibrahim ${ }^{1}$, Hala E I Jubara ${ }^{2}$ \\ ${ }^{1}$ Alnielian University, Department of Engineering, Khartoum, Sudan
}

\begin{abstract}
In this paper we will study the architecture of the Long Term Evolution LTE of Universal Mobile Telecommunication System UMTS with Multi User Multiple Input Multiple Output MU-MIMO technique. Open-Loop Spatial Multiplexing OLSM is one of nine transmission modes in LTE networks, in OLSM transmissions are configured with minimal feedback from the UE; we will consider OLSM in this study while performing three different types of scheduling algorithms that are used in LTE. The implementation of this study will use MATLAB software with LTE system level simulation, and results will collected for UE's throughputs and compared with each other for the different types of schedulers and with different antenna configurations such as $2 x 2,2 x 4$, and $4 x 4$.
\end{abstract}

Keywords: LTE, Scheduling, OLSM, MU-MIMO, RR, PF, Best CQI.

\section{Introduction}

LTE standard has been published by $3 \mathrm{GPP}$ as an extension of UMTS (based on 3GPP standard) and 1xEV-DO (base on 3GPP2 standard) technologies. LTE is mainly designed for high speed data applications both in the uplink and downlink. LTE network offers about $300 \mathrm{Mbps}$ data rate in the downlink and about $75 \mathrm{Mbps}$ in the uplink. There is possibility of supporting voice over LTE (VoLTE) in the future. There are various methods under progress to support VoLTE some of them includes VOIP, legacy fall back to previous existing wireless networks [1].

\section{Literature Review}

The Air interface between LTE eNodeB and UE supports high data rate owing to OFDM and multiple antenna techniques employed. OFDMA is used from network to UE air interface and SC-FDMA is used from UE to network air interface. Refer following links to know OFDMA basics.

\subsection{LTE System Architecture Evolution}

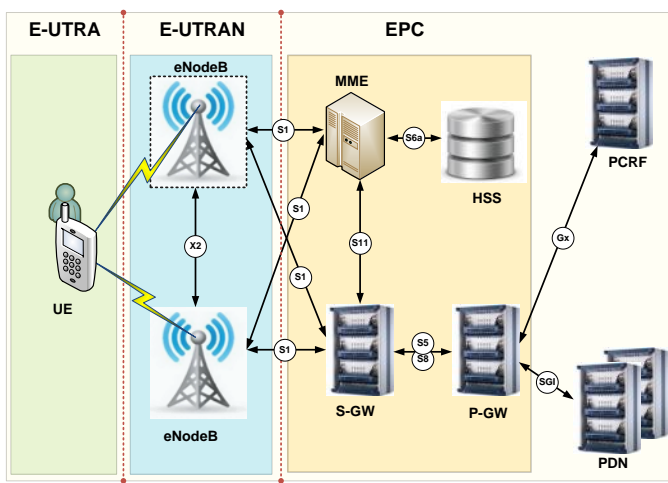

Figure 1: LTE architecture

As shown in the figure LTE SAE (System Architecture Evolution) consists UE, eNodeB and EPC(evolved packet core). Various interfaces are designed between these entities which include Uu between UE and eNodeB, X2 between two
eNodeB, S1 between EPC and eNodeB. eNodeB has functionalities of both RNC and NodeB as per previous UMTS architecture. LTE is completely IP based network. The basic architecture contains the following network elements [1].

1) LTE EUTRAN (Evolved Universal Terrestrial Radio)

2) LTE Evolved Packet Core.

\subsection{Downlink Scheduling in LTE}

The downlink physical resource is considered as a timefrequency resource grid consisting of multiple resource blocks (RB). A resource block is divided in multiple resource elements (RE). A scheduler is a key element in the eNB and it assigns the time and frequency resources to different users in the cell [15]. Thus a RB is the smallest element that can be assigned by the scheduler. Our research is focused on the Round Robin (RR), proportional Fair (PF), and on the Best CQI scheduling algorithms.

In RR scheduling the terminals are assigned the resource blocks in turn (one after another) without taking the Channel Quality Indication (CQI) into account. Thus the terminals are equally scheduled. The PF scheduling algorithm provides a good trade-off between system throughput and fairness by selecting the user with highest instantaneous data rate relative to its average data rate. The Best CQI scheduling assigns the resource blocks to the user with the highest CQI on that RB. To perform Best CQI scheduling the UE must feedback the (CQI) to the BS

\subsubsection{Round Robin scheduling}

The scheduler assigns resources cyclically to the users without taking channel conditions into account. This is a simple procedure giving the best fairness as shown the flowchart below in figure (2). But it would offer poor performance in terms of cell throughput. 


\section{International Journal of Science and Research (IJSR) \\ ISSN (Online): 2319-7064}

Index Copernicus Value (2013): 6.14 | Impact Factor (2015): 6.391

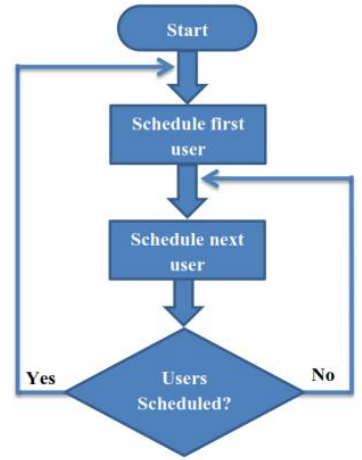

Figure 2: Round robin scheduling flowchart

\subsubsection{Proportional Fair scheduling}

The scheduler can exercise Proportional Fair (PF) scheduling allocating more resources to a user with relatively better channel quality. This offers high cell throughput as well as fairness satisfactorily. Thus, Proportional Fair (PF) scheduling may be the best option. Proportional fair scheduling algorithm can be represented as the flow chart below of figure (3).

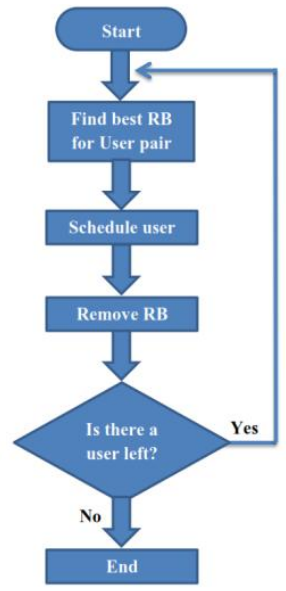

Figure 3: Proportional fair scheduling flowchart

\subsubsection{Best CQI scheduling}

The scheduler allocates resources to those users with best channel condition. For this, users should send CQI feedback to eNodeB. The highest CQI value means best channel conditions and the recourses will allocate to this users. In this algorithm the users who are far away from eNodeB (i.e. Cell edge users) don't schedules well. The Best CQI scheduling flowchart is shown in figure (4) below.

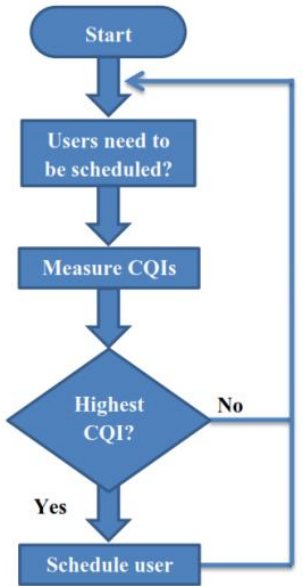

Figure 4: Best CQI scheduling flowchart

\section{System Model}

For efficient deployment of LTE, performance analyses of different radio parameters are worth evaluating. Simulations are necessary to test and optimize algorithms and procedures. These have to be carried out on both the physical layer and in the network context. LTE physical layer is important for conveying both data and control information between an eNodeB and UE. To enable reproducibility of our results, simulation of the network is done by using a system level simulator [9, 11-14].

The system level simulator follows a standard way in order to get the specified results from the predefined parameters by applying the structured procedures, protocols, and algorithms that are legally certified by the 3GPP LTE project standard development technical specifications. To generate the specified results an extending work in the system level simulator is achieved. The block diagram below describes the general structure of the methodology that followed in this study.

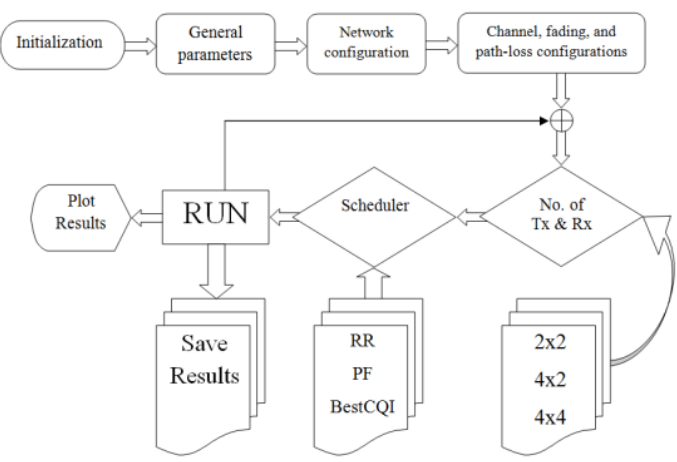

Figure 5: Methodology General Structure

According to the above strategy that been followed in this study, a cross comparison of six loops is executed simultaneously with different multipath transmissions and schedulers.

\section{Simulation Description}

Because of that, transmission mode of adaptation technology based networks can be adapted semi statically by dynamic resource scheduling controls according to various channel conditions that occurs. Spatial multiplexing transmission modes allow multiple antennas to send and receive multiple parallel streams. So that sometimes referred to as the true MIMO technique. One of the Spatial multiplexing transmission modes is the OLSM which referred to by (transmission mode 3 ). In the OLSM the UE estimates the radio channel with minimum feedback required.

Initialization step parameters is defined as setting the default parameters that used to calculate the general configuration variables, these variables are calculated below from the table (1) of the simulation default parameters. 


\section{International Journal of Science and Research (IJSR) \\ ISSN (Online): 2319-7064}

Index Copernicus Value (2013): 6.14 | Impact Factor (2015): 6.391

Table 1: Simulation default parameters

\begin{tabular}{|c|c|}
\hline Parameter & value \\
\hline subcarrier spacing & $15 \mathrm{KHz}$ \\
\hline TTI length & 0.001 second $=1 \mathrm{~ms}$ \\
\hline RB bandwidth & $180 \mathrm{KHz}$ \\
\hline code word per TTI & 2 \\
\hline number of RB in $10 \mathrm{MHz}$ & 50 \\
\hline IFFT(Tx) /FFT size $(\mathrm{Rx})$ & 1024 \\
\hline Cyclic prefix & normal \\
\hline No. of symbols $\left(\mathrm{N}_{S y m}\right)$ & 7 \\
\hline Symbol length & 72 samples \\
\hline
\end{tabular}

Generally, the system network consist of two eNodeB rings which includes 57 eNodeB represents 19 sites, only 21 eNodeB will be taken into account for results. CLSM spatial multiplexing will likely be the transmission mode for system model based on this study. All parameters that considered in the implementation of the system model in study are specified as in the table (2) below.

Table 2: Simulation Parameters

\begin{tabular}{|c|c|}
\hline \multicolumn{2}{|c|}{ General parameter } \\
\hline Frequency & $2.14 \mathrm{GHz}$ \\
\hline Bandwidth & $10 \mathrm{MHz}$ \\
\hline Transmission mode & Mode 3, OLSM \\
\hline No. of $\mathrm{Tx} \mathrm{Rx}$ & $2 \times 2,4 \times 2,4 \times 4$ \\
\hline Simulation time & 10 TTI \\
\hline SINR averaging algorithm & MIESM \\
\hline Receiver type & ZF (Zero Forcing) \\
\hline \multicolumn{2}{|c|}{ Network parameters } \\
\hline Number of eNodeB rings & 2 \\
\hline Distance between eNodeBs & 500 meter \\
\hline eNodeB's transmit power & $40 \mathrm{~W}$ \\
\hline Number of UE per eNodeB & 5 \\
\hline UE speed & $5 / 3.6=1.38 \mathrm{~m} / \mathrm{s}=5 \mathrm{Km} / \mathrm{h}$ \\
\hline \multicolumn{2}{|c|}{ Path- loss parameters } \\
\hline Path-loss environment & Urban TS-36.942 \\
\hline Minimum coupling loss & $70 \mathrm{~dB}$ \\
\hline \multicolumn{2}{|c|}{ Shadow fading parameters } \\
\hline Channel model type & Winner II [1] with correlated fading \\
\hline \multicolumn{2}{|c|}{ eNodeB parameters } \\
\hline Antenna gain pattern type & Kathrein Antenna "742212" \\
\hline Max antenna gain & $\begin{array}{l}15 \text { recommended for urban area } \\
(2000 \mathrm{MHz})\end{array}$ \\
\hline Site height & $20 \mathrm{~m}$ \\
\hline Antenna frequency & $2140 \mathrm{MHz}=2.14 \mathrm{GHz}$ \\
\hline \multicolumn{2}{|c|}{ UE parameters } \\
\hline Receiver noise figure & $9 \mathrm{~dB}$ \\
\hline Thermal noise density & $-174 \mathrm{dBm} / \mathrm{Hz}$ typical value \\
\hline Receiver height & $1.5 \mathrm{~m}$ \\
\hline
\end{tabular}

Simulation parameters that expressed above are considered as the assumption in the Evolved Universal Terrestrial Radio Access (E-UTRA); Radio Frequency (RF) system scenarios technical specification documents and applied to the system level simulator using the methodology in figure 8 .

From table 2, the Minimum coupling loss describes the minimum loss in signal $[\mathrm{dB}]$ between BS and UE or UE and $\mathrm{UE}$ in the worst case. And it is defined as the minimum distance loss including antenna gains measured between antenna connectors. Recommended values are $70 \mathrm{~dB}$ for urban areas, $80 \mathrm{~dB}$ for rural. Also Path-loss model specified as recommended for macro cell propagation model below.

\subsection{Channel model}

Winner II MIMO radio channel model enables system level simulations and testing. This means that multiple links are to be simulated (evolved) simultaneously. Antenna frequency is the frequency at which the pattern should be used $[\mathrm{MHz}]$. It is not automatically set to the frequency being used because it could happen that all you are interested is the pattern itself, not whether it would correspond with the actual frequency used.

The 40 watts eNodeB transmit power chosen as recommended for urban areas, which equal to $46.0206 \mathrm{dBm}$ by using the equation:

$$
y=10 \times \log _{10} u+30, \text { where } u=40 \mathrm{~W}
$$

\subsection{SINR averaging}

The effective SINR value in [32] is calculated as a compression from a set of quality measures of resource elements (in OFDMA transmission quality measures are instantaneous subcarrier SINR samples). The effective SINR mapping approach maps the instantaneous channel state characterized by $\mathrm{N}$ quality measures of time, frequency and/or space resource elements into an instantaneous effective channel quality measure. Afterwards, effective channel quality measure is mapped to the performance metric, such as Block Error Rate (BLER). The approximated maximal UE data capacity is calculated in bits per second as follow:

$$
C_{\max }=B W \times C R_{\text {eff }} \times(1-B L E R)
$$

Where BW is the total bandwidth offered by LTE, $C R_{\mathbb{a}}$ is the effective code rate of the selected modulation scheme.

\subsection{Performance metrics}

Fairness rates how equally a resource (in this case throughput) is distributed over $\mathrm{N}$ users, and is defined in [24] as:

$$
J(x)=\frac{\left(\sum_{i=1}^{N} x_{i}\right)^{2}}{N \sum_{i=1}^{N} x_{i}^{2}}, \text { Whe: }
$$

( ) is a vector of length $\mathrm{N}$, containing the throughput of each user from the number of users that need to be simulated. The value of $f($ varies from 1 to 0 indicating decreasing order of fairness and is inversely proportional to throughputs.

An Empirical cumulative distribution function (ECDF) with a pendent of one and a flat SINR-to-throughput plot, while a minimum fairness would produce a step function ECDF and a single non-zero throughput point in the SINR to throughput plot.

\section{Results and Discussion}

Here the simulation results is gathered and discussed in terms of throughput of each scheduler, and the throughput per each UE, and the peak, mean, and edge throughput, which are the available statistics that provided from the 


\section{International Journal of Science and Research (IJSR) \\ ISSN (Online): 2319-7064}

Index Copernicus Value (2013): 6.14 | Impact Factor (2015): 6.391

system level simulator.

In figure (11) result shows the UE throughput ECDF distribution curve for $2 \times 2$ antenna configuration, which shows that the RR and PF has accumulative distribution with continuous increment as the increment of the general throughput, while the best CQI scheduler has an average UE throughput distribution curve.

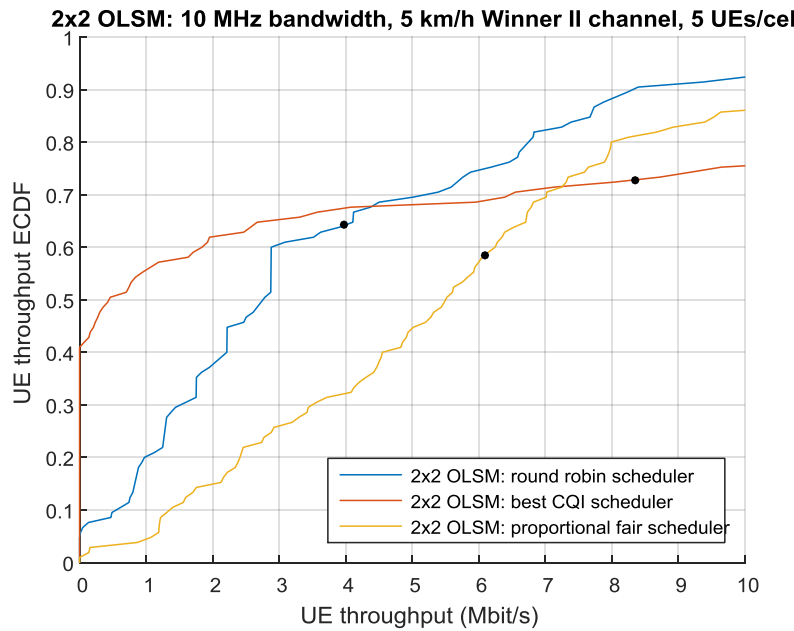

Figure 11: UE throughput ECDF (2x2)

For general throughput results and fairness index for $2 \times 2$ antenna configuration shown below in figure (12) and the results show that the best CQI scheduler has the maximum throughput as recorded (8.4 Mbps mean throughput and 47.5 Mbps peak throughput) but with minimum fairness value. The proportional fair scheduler has advantage over the RR scheduler and it also has higher fairness value, the RR scheduler records the minimum throughput values because it doesn't depends on anything the scheduler just schedule the users circularly one after another.

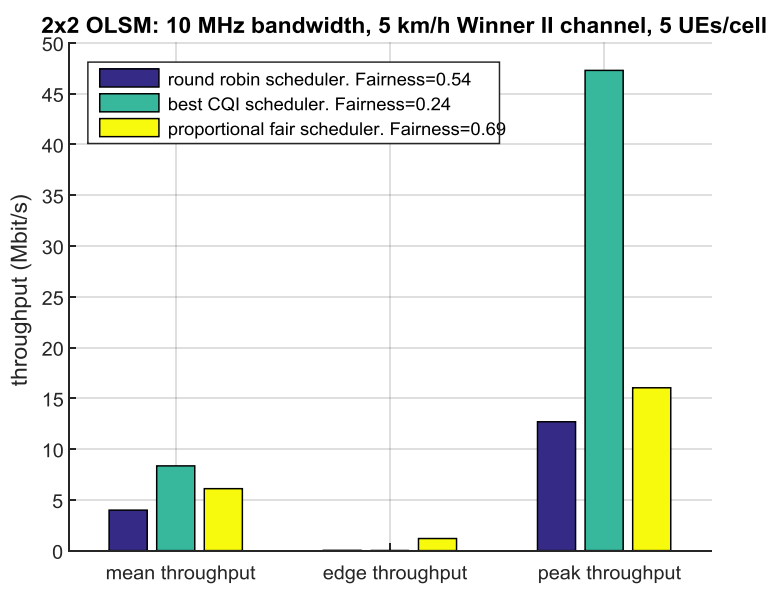

Figure 12: mean, edge, and peak throughput, and fairness (2x2)

The figure (13) shows the UE throughput ECDF distribution curve for $4 \times 2$ antenna configurations, here the RR and the PF schedulers has approximately the same distribution curves, while the best CQI shows an average distribution of throughput per UE.

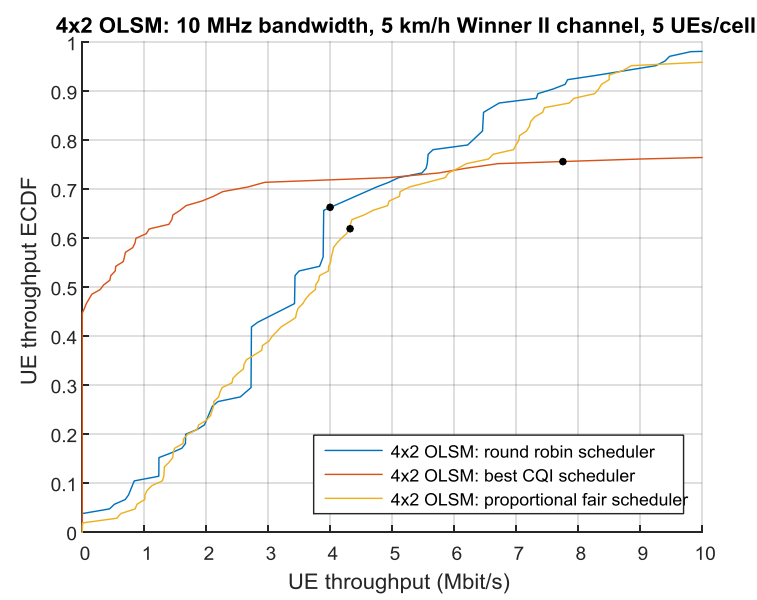

Figure 13: UE throughput ECDF $(4 \times 2)$

Here the general throughput for $(4 \times 2)$ shown in figure (14) and the fairness results, the result is similar to $(2 \times 2)$ results but with some different, is that here the $\mathrm{RR}$ and $\mathrm{PF}$ schedulers has the same throughput results, and fairness values.

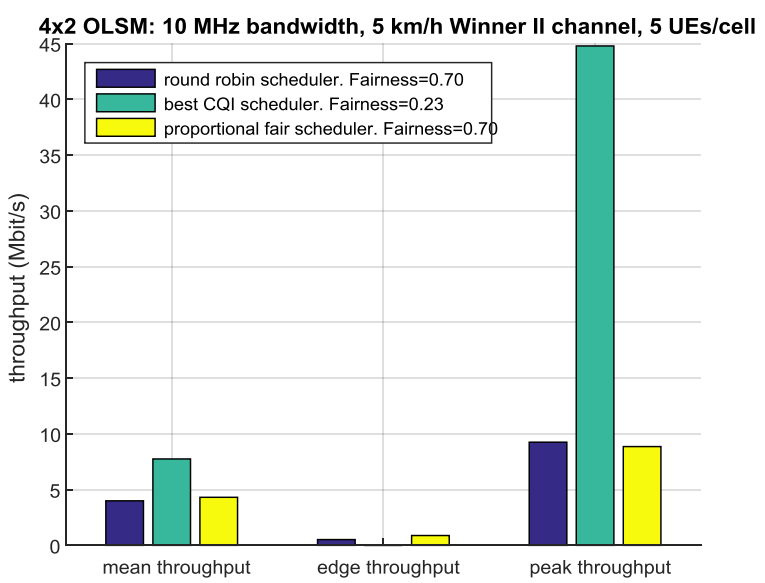

Figure 14: mean, edge, and peak throughput, and fairness $(4 \times 2)$

The figure (15) shows the UE throughput ECDF distribution curve for $4 \times 4$ antenna configurations, here the Best CQI has an obvious advantage over the other scheduler in term of throughput distribution over UEs, while the performance of the RR and PF is degraded.

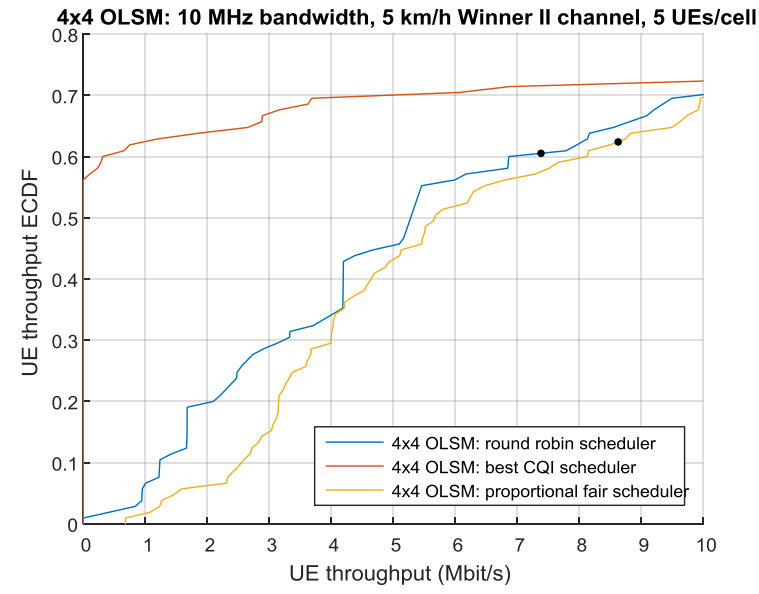

Figure 15: UE throughput ECDF $(4 \times 4)$

\section{Volume 5 Issue 6, June 2016 www.ijsr.net}




\section{International Journal of Science and Research (IJSR) \\ ISSN (Online): 2319-7064}

Index Copernicus Value (2013): 6.14 | Impact Factor (2015): 6.391

For general throughput results and fairness index for $4 \times 4$ antenna configuration shown below in figure (16) and the results show that the best CQI scheduler still has the maximum throughput as recorded $(\sim 18$ Mbps mean throughput and $\sim 109$ Mbps peak throughput) but also with minimum fairness value and in all cases the best CQI has no edge throughput records. The proportional fair scheduler and the RR scheduler has also approximately similar results in term of throughput and fairness.

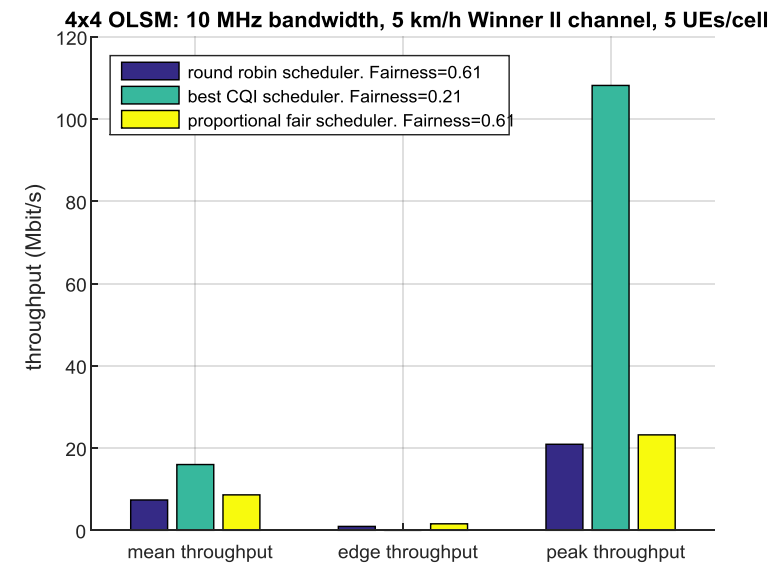

Figure 16: mean, edge, and peak throughput, and fairness $(4 \times 4)$

\section{Conclusion}

Scheduling techniques is one of the necessary parameters LTE downlink which controls that how the users will be served and how they will be satisfied. In this study we perform a comparison study between three major schedulers in LTE, which are the RR, PF, and the Best CQI schedulers. The comparison performance metrics are depending on the terms of throughput and distribution of throughput among users and fairness value.

The Best CQI scheduler has the overall advantage than other in term of mean and peak throughput, but it also has a minimum fairness records which is expected according to it dependent nature, but also to be mentioned that the best CQI has poor distribution of throughput per UEs in $(2 \times 2$ and $4 \times 2)$ antenna configuration, but with $(4 \times 4)$ it has the best distribution throughput over UEs curve.

The Proportional Fair scheduler has higher throughput and fairness over the Round Robin in $(2 \times 2)$ antenna configuration, but with the increment of number of transmitters and receivers the RR and PF has approximately similar results.

\section{References}

[1] Pierre Lescuyer, Thierry Lucidarme, "Evolved packet system (EPS): the LTE and SAE evolution of 3G UMTS", John Wiley \& Sons, Ltd.

[2] Qinlong Qiu, Dongmei Zhang, Jian Ma, "GPRS network model in NS 2", in proceedings of IEEE The Joint Conference of 10th Asia-Pacific Conference on
Communications and 8th International Symposium on Multi-Dimensional Mobile Communications,

[3] 3GPP TS 36.300, 3rd Generation Partnership Project; Technical Specification Group Radio Access Network; Evolved Universal Terrestrial Radio Access (E-UTRA) and Evolved Universal Terrestrial Radio Access Network (E-UTRAN);Overall description; Stage 2 (Release 12).

[4] Hossam S. Hassanein, Abd-Elhamid M. Taha, Najah Abu Ali (2012)."LTE, LTE-advanced, and WiMAX : towards IMT-advanced networks", John Wiley \& Sons, Ltd.

[5] L. Hanzo, J. Akhtman, L. Wang, M. Jiang (2011) MIMO-OFDM for LTE, WiFi, and WiMAX : coherent versus non-coherent and cooperative turbo-transceivers , 1st edn., The Atrium, Southern Gate, Chichester, West Sussex, PO19 8SQ, United Kingdom: John Wiley \& Sons, Ltd.

[6] 3GPP TS 36.212: "Evolved Universal Terrestrial Radio Access (E-UTRA); Multiplexing and channel coding".

[7] 3GPP TS 36.201 "Evolved Universal Terrestrial Radio Access (E-UTRA); LTE physical layer; General description".

[8] Y. Zaki, T. Weerawardane, C. Gorg, and A. Timm-Giel, "Multi-QoS-Aware Fair Scheduling for LTE", IEEE Veh. Tech. Conf., VTC-Spring, May 2011.

[9] M. Iturralde, A. Wei, and A.Beylot, "Resource Allocation for Real Time Services Using Cooperative

Game Theory and a Virtual TokenMechanism in LTE Networks," in Proc. IEEE Personal Indoor Mobile Radio Commun., PIMRC, Sydney, Australia, Jan. 2012.

[10] K. Sandrasegaran, H. A. Mohd Ramli, and R. Basukala, "Delay-Prioritized Scheduling (DPS) for Real Time Traffic in 3GPP LTE System," in Proc. IEEE Wireless Commun. And Net. Conf., WCNC, Apr. 2010.

[11] G. Monghal, D. Laselva, P. Michaelsen, and J. Wigard, "Dynamic Packet Scheduling for Traffic Mixes of Best Effort and VoIP Users in E-UTRAN Downlink," in Proc. IEEE Veh. Tech. Conf., VTC-Spring, Marina Bay, Singapore, May 2010.

[12] S. Choi, K. Jun, Y. Shin, S. Kang, and V. Lau, "MAC Scheduling Scheme for VoIP Traffic Service in 3G LTE," in Proc. IEEE Veh. Tech. Conf., VTC-Fall, Baltimore, MD, USA, Oct. 2007

[13] G.Mongha, K.I. Pedersen, I.Z. Kovacs, P.E. Mogensen, " QoS Oriented Time and Frequency Domain Packet Schedulers for The UTRAN Long Term Evolution", In Proc. IEEE VTC, 2008

[14] F. Capozzi, G. Piro, L.A. Grieco, G. Boggia, P. Camarda, "Downlink Packet Scheduling in LTE Cellular Networks: Key Design Issues and Survey", International Journal of Information and Electronics Engineering, Vol. 5, No. 3, 2015.

[15] Mohammad T. Kawser, Hasib M. A. B. Farid, Abduhu R. Hasin, Adil M. J. Sadik, and Ibrahim K. Razu, "Performance Comparison between Round Robin and Proportional Fair Scheduling Methods for LTE", International Journal of Information and Electronics Engineering, Vol. 2, No. 5, September 2012.

[16] B. Sahoo, "Performance Comparison of Packet Scheduling Algorithms for Video Traffic in LTE Cellular Network", International Journal of Mobile 


\section{International Journal of Science and Research (IJSR) \\ ISSN (Online): 2319-7064}

Index Copernicus Value (2013): 6.14 | Impact Factor (2015): 6.391

Network Communications \& Telematics ( IJMNCT) Vol. 3, No.3, PP 09-18, June 2013.

[17] Vallari Sharma, Prof. P.K Chopra, 2014 "A Survey on LTE Downlink Packet Scheduling", International Journal of Advanced Research in Computer and Communication Engineering, Vol. 3, Issue 9.

[18] Davinder Singh, Preeti Singh, June 2013 "Radio Resource Scheduling in 3GPP LTE: A Review", International Journal of Engineering Trends and Technology (IJETT), Volume 4 Issue 6.

[19] A.Biernacki, K.Tutschku, 2013 "Comparative Performance Study of LTE Downlink Schedulers", Springer, Vol.74, No.2, pp 585-599.

[20] B.Lui, H.Tang, L.Xu , 2013“ An Efficient Downlink Packet Scheduling Algorithm for Real Time Traffics in LTE Systems", IEEE CCNC, Vol.44, No.3, pp 1-5.

[21] H. Al-Jaradat, K. Sandrasegaran, 2013 "On the Performance of PF, MLWDF and EXP/PF algorithms in LTE”, IJCT , Vol8, No.1, pp 132-142.

[22] J. C. Ikuno, M. Wrulich, and M. Rupp, System level simulation of LTE networks, in Proc. 2010 IEEE 71st Vehicular Technology Conference, Taipei, Taiwan, May 2010. [Online] Avaialable: http://publik.tuwien.ac.at/files/PubDat_184908.pdf

[23] C. Mehlfuhrer, M. Wrulich, J. C. Ikuno, D. Bosanska, and M. Rupp, "Simulating the long term evolution physical layer," in Proc. the 17th European Signal Processing Conference (EUSIPCO 2009), Aug. 2009, pp. 1471-1478.

[24] J. C. Ikuno, M. Wrulich, and M. Rupp, "System level simulation of LTE networks," in Proc. IEEE 71st Vehicular Technology Conference (VTC-Spring), May 2010, pp. $1-5$.

Volume 5 Issue 6, June 2016 www.ijsr.net 